\title{
Proton nuclear magnetic resonance spectroscopic detection of oligomannosidic $n$ glycans in alpha-mannosidosis: a method of monitoring treatment
}

\author{
Derk Frederik Matthaus Avenarius • \\ John-Sigurd Svendsen • Dag Malm
}

Received: 19 December 2010 /Revised: 22 March 2011 / Accepted: 31 March 2011 /Published online: 4 May 2011

(C) The Author(s) 2011. This article is published with open access at Springerlink.com

\begin{abstract}
In Alpha-mannosidosis (MIM 248500) the patients accumulate mainly unbranched oligosaccharide chains in the lysosomes in all body tissues, including the brain. With ensuing therapeutic modalities in man (BMT and ERT) non-invasive methods of monitoring the effect of treatment are needed. Paramount is the possible effect of the treatment on the brain, since this organ is regarded as difficult to reach because of the blood-brain barrier. We therefore performed proton nuclear magnetic resonance spectroscopy (MRS) of the brain in two untreated patients, and a 16-year-old patient treated with BMT at the age of 10 to assess whether this non-invasive method could be applied in the monitoring of the accumulation of abnormal chemicals in the brain of patients. We found an abnormal peak that was not present in the treated patient. A similar pattern was also found in MRS of urine from patients, reflecting the concentration of oligosaccharides in serum and tissues. We therefore conclude that MRS can be a useful method to monitor the effect of treatment for Alpha-Mannosidosis.
\end{abstract}

\section{Introduction}

Alpha-Mannosidosis (MIM 248500) is an autosomal recessive lysosomal storage disorder resulting from deficient activity of lysosomal alpha-mannosidase. Affected patients accumulate mainly unbranched oligosaccharide chains in the lysosomes, of which the major storage

Communicated by: Ed Wraith

Competing interest: None declared.

D. F. M. Avenarius $(\bowtie) \cdot$ J.-S. Svendsen $\cdot$ D. Malm

University Hospital of Northern-Norway,

Tromso, Norway

e-mail: derk.avenarius@unn.no product is the trisaccharide Man $\alpha 1-3$ Man $\beta 1-4$ GlcNAc (Michalski et al. 1990). Patients accumulate mannose-rich oligosaccharides in serum and most tissues. They suffer from mental retardation, immune deficiency and skeletal deformities (Øckermann 1967).

In parallel with the management of Gaucher disease with enzyme replacement therapy (ERT), several studies to investigate the effect of ERT in mannosidosis with recombinant enzyme have been performed in mice (Stinchi et al. 1999) or in guinea pigs (Berg and Hopwood 2002). The effect of the treatment on cerebral and extra-cerebral functions has been assessed by studying histology in tissues after sacrificing the animals (Roces et al. 2004; D'Hooge et al. 2005). In addition bone marrow transplants (BMT) have been performed in cats (Walkley et al. 1994) and man (Wall et al 1998). BMT has been considered a likely success, although the number of patients is small and measured parameters "soft" (Grewal et al. 2004). With ensuing therapeutic modalities in man (BMT and ERT), noninvasive methods of monitoring the effect of treatment are needed. Paramount is the possible effect of the treatment on the brain, since this organ is regarded difficult to reach because of the blood-brain barrier (Johanson et al. 2011).

We therefore performed proton magnetic nuclear resonance spectroscopy of the brain in two untreated patients, and a 16-year-old patient treated with BMT at the age of 10, to test whether this non-invasive method could be applied in the monitoring of the accumulation of abnormal chemicals in the brain of patients.

In MR imaging, one uses the signal from water and fat protons to create an image. MRS is a method of detecting certain metabolites that are not used in normal MR imaging due to their low concentrations and the difference in their resonance frequencies from water and fat. In single voxel MRS all the signal coming from one defined volume is 
displayed as a single curve. Different metabolites are then displayed as different peaks on their specific resonance frequencies. These frequencies are expressed as parts per million (ppm) differences from a standard. A typical substance like $\mathrm{N}$-acetylaspartate (NAA) will thus always be displayed at $2.0 \mathrm{ppm}$. This makes it possible to identify the different molecules. The relative amount of signal is displayed as the peak height, and its relation to other metabolites is studied with peak height ratios calculation. For these calculations an internal reference (the Creatine peak height at $3 \mathrm{ppm}$ ) is often used since this signal has been shown to be relatively stable. For absolute quantification of the MRS signal, one could use an external reference but this is normally not done. Modern MR Scanners are now able to perform an MRS scan within a few minutes with automatic data analysis, making it possible to examine many patients without the need for sedation. It is now part of routine scanning in many institutions. However many younger patients will often not be able to comply with prolonged scanning times and would still need sedation.

\section{Methods}

In two untreated patients (age 36 and age 20) and one BMT treated patient (age 16) routine unsedated MR imaging was supplied with single voxel proton spectroscopy on a 1.5 Tesla whole body Philips scanner. All examinations were performed after informed consent was obtained from the subjects or their parents. Single voxel proton spectroscopy was performed with short $22-30 \mathrm{~ms}$ and intermediate $136 \mathrm{~ms}$ and longer echo-times with PRESS technique, in the basal ganglia, periventricular white matter and occipital grey matter. Due to relatively long examination times for spectroscopy not all areas were examined in all patients.

Fasting early morning urine samples from the BMT treated patient and from an untreated patient were examined with in vitro spectroscopy to verify the in vivo findings. The in vitro spectroscopy was performed on a Varian Inova NMR spectrometer with field strength of 14 Tesla, using PRESAT water suppression.

Our patients displayed a uniform disease presentation that started in the first year of life and which included immune deficiency, hearing deficits, mental retardation, and skeletal deformities. One patient was treated with BMT at age 10; with the engraftment proven to be $100 \%$ one year later.

\section{Results}

The MR imaging done prior to the spectroscopy uptake did not show any focal lesions. One patient had a narrow foramen magnum and some tonsillar descent.
Representative proton spectra from a voxel placed in the basal ganglia, with short echo times are shown in Fig. 1.

The analysis of the spectra is summarized in Table 1.

The short echo times spectra show a broad elevation of the peaks around $3.7 \mathrm{ppm}$ as well as some increase of the
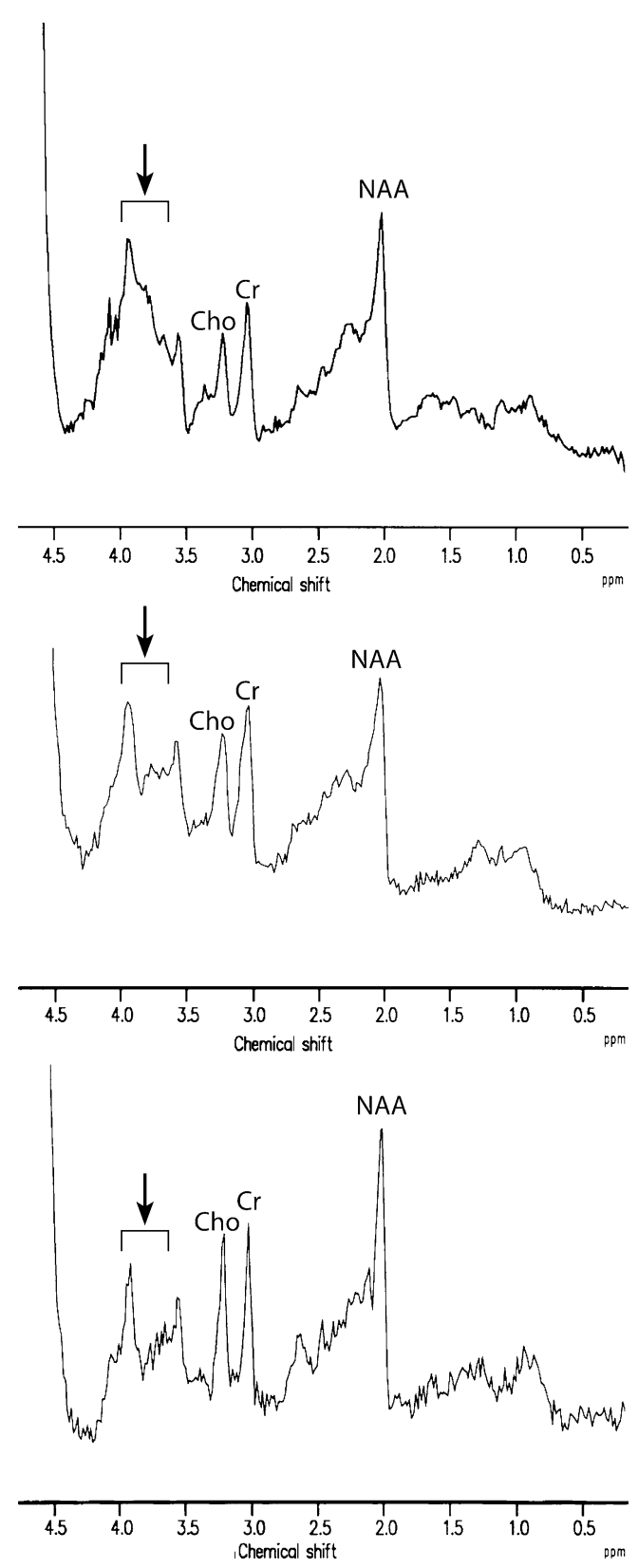

Fig. 1 Upper spectrum: short echo-time proton spectroscopy of a 20 year old untreated patient taken in the region of the basal ganglia, with a broad elevation around $3.7 \mathrm{ppm}$.(arrow). Middle spectrum: short echo-time proton spectroscopy of a BMT treated patient taken in the region of the basal ganglia, with a mild broad elevation around $3.7 \mathrm{ppm}$ (arrow) and somewhat elevated second Creatine peak at $3.9 \mathrm{ppm}$. Lower spectrum: Short echo-time proton spectroscopy of a control subject showing a normal spectrum, with normal amount of signal where our patients showed increased signal (arrow) 
Table 1 Analysis of the spectra

\begin{tabular}{|c|c|c|c|c|c|c|c|}
\hline & Agelsex & Treatment & Location & $\mathrm{NAA} \backslash \mathrm{Cr}$ & CholCr & $\mathrm{mI} \backslash \mathrm{Cr}$ & $\mathrm{pOS} \backslash \mathrm{Cr}$ \\
\hline Patient 1 & $16 \backslash \mathrm{F}$ & BMT & BG & 1.2 & 0.9 & 0.8 & 1.0 \\
\hline \multirow[t]{2}{*}{ Patient 2} & $20 \backslash \mathrm{F}$ & none & BG & 1.6 & 0.8 & 0.8 & 1.4 \\
\hline & & & WM & 1.8 & 0.9 & NA & 1.6 \\
\hline Patient 3 & $36 \backslash F$ & none & OGM & 1.8 & 0.6 & 0.8 & 0.9 \\
\hline Normal values & & & & $1.1-1.8$ & $0.5-0.9$ & $0.5-0.7$ & $0.5-0.7$ \\
\hline
\end{tabular}

Abbreviations: BG, basal ganglia; WM, white matter; OGM, occipital grey matter; pOS presumptive oligosaccharides. Normal values taken from: Danielsen ER, Ross B (1999) Magnetic resonance spectroscopy diagnosis of neurological diseases. Chapter 2 Basic Physics of MRS.

second Creatine peak at 3.9 ppm(Fig. 1). This was most pronounced in the untreated patients but still visible in the BMT treated patient. The pathological increase of signal in our patients disappeared at intermediate and long echotimes (136 ms and longer), and spectra obtained with these echo times had normalized (not shown).

In vitro spectroscopy of urine samples from an untreated and a BMT treated patient show extra peaks from 3.6 to 3.8 ppm most probably representing the same oligosaccharides (Fig. 2).
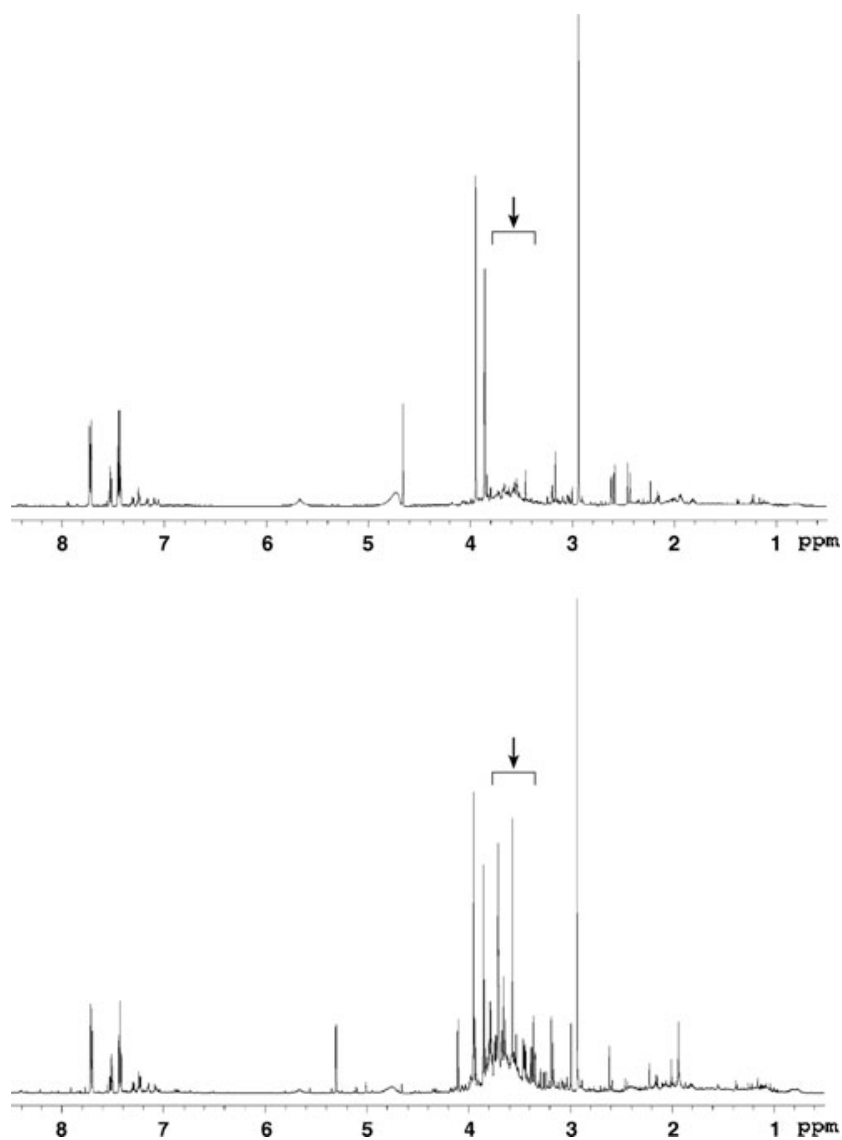

Fig. 2 In vitro spectroscopy scan of urine samples from a BMT treated patient(upper spectrum) and from a untreated patient(bottom spectrum), showing additional peaks in the untreated patient from 3.57 to $3.79 \mathrm{ppm}$ (arrow) representing the oligosaccharides

\section{Discussion}

Ever since the introduction of magnetic resonance, the brain of patients with Alpha-Mannosidosis and other lysosomal storage diseases has been studied, first with morphologic imaging (Patlas et al. 2001; Autti et al. 2007; Seto et al. 2001) and later with functional MR imaging such as diffusion weighting (Patay 2005). These imaging methods have the potential to show the damage or developmental disturbances due to these diseases, but it is only by MRS one can attempt to measure the amount of accumulated oligosaccharides and other molecules. Functional neuron distribution of $\mathrm{N}$-acetylaspartate (NAA) was studied in 60 patients with Mucopolysaccharidosis (MPS) and was shown to be decreased in lesions visible on standard imaging (Takahashi et al. 2001; Vendolin et al. 2007), whereas Myo-inositol (3.6 ppm) a marker of gliosis, was found to be increased within lesions. A more recent study of a cat model of alpha-mannosidosis (Magnitsky et al. 2010) examined the brain of normal and affected cats with high field (4.7 Tesla) MRS. They showed that the MRS spectra had the same properties as those spectra in MPS patients with broad high peaks at $3.7 \mathrm{ppm}$. They could also distinguish an additional smaller peak at around $2 \mathrm{ppm}$.

The increase in signal in our Mannosidosis patients is best shown on short echo-time spectra due to the short relaxation times of the metabolites. A short relaxation time means that the signal disappears faster than other metabolites. At these longer echo-times most of the oligosaccharides as well as glutamate, glutamine, lipids, and myoinositol will not show any signal. In clinical practice, when studying diseases related to molecules with short relaxation times, only short echo time spectra are considered, as we have done here. To describe eventual abnormalities, an internal reference is used $(\mathrm{Cr})$ and peak to peak ratios or areas are calculated and compared. These ratios are only measurable in clinical practice on clearly defined non overlapping peaks. Our patients showed broad areas of elevation centred at $3.7 \mathrm{ppm}$, overlapping other metabolites like Myo-inositol at $3.6 \mathrm{ppm}$, Alpha glutamine and 
glutamate at 3.6-3.8 and the second Creatine peak at 3.9, making exact quantification and peak to peak ratio calculations difficult. Results of these measurements are shown in Table 1. The range of normal values for NAA is large in the area of the basal ganglia, the NAAICr ranges from 1.1 in the putamen to 1.8 in white matter. The additional peak at $2 \mathrm{ppm}$ that was seen in cats at high field MR was not clearly seen in our patient (Fig. 1) but the NAA peak shows some broadening here that could be related to this peak. Further research with higher field strength is needed to assess this peak. The MRS signal in our BMT treated patient (Fig. 1) was almost completely normal which is consistent with the MRS spectra findings in BMT treated MPS patients (Takahashi et al. 2001). The broadness of the spectrum due to the different resonances of the oligosaccharides is also reflected in the urine sample high field spectroscopy where multiple peaks are observed at the same resonance frequencies as the in vivo spectra (Fig. 2).

To our knowledge, this is the first time that MRS is performed in an Alfa-Mannosidosis patient treated with BMT. We found a strong pathological signal in an untreated patient and an almost normal spectrum in the BMT treated patient. We have demonstrated that the Mucopolysaccharidosis and Oligosaccharidosis patients appear to have abnormal peaks in the same position on the spectra, and that short echo time MRS can be used to detect this signal. The ratio of the pathological signal in MPS patients outside lesions has been reported to range from 0.6-1.5 (Takahashi et al. 2001), and our range was from $0.9-1.6$. (Table 1 ).

MRS was performed on three Alfa-Mannosidosis patients by Gutschalk et al. 2004, and they did not report any additional peaks. The actual spectra taken are not shown in their article, but short echo times and automatic post processing were used and one would therefore expect to find a pathological peak. One explanation could be that they examined patients with the rare adult variant of the disease and that these patients have a higher residual enzyme capacity and therefore a reduced accumulation of metabolites. Another explanation could be that the patient group was older (3847 years). It is possible that the biochemical environment or state of the metabolites we are attempting to detect changes over time and that the MRS features also change. One of our patients was 36 years of age and we obtained only one spectrum from occipital grey matter (not shown), and although there was a clearly pathological peak its maximum peak height was lower than would be expected in an individual that had more time to accumulate oligossacharides. This finding could be consistent with the findings of Gutschalk et al. 2004. It would be necessary to examine patients over time to see if there exists such an age related pattern.

\section{Conclusion}

This study demonstrates that MRS can detect peaks consistent with carbohydrate moieties in the brain of patients with Alpha-Mannosidosis. In the BMT treated patient, 6 years post transplant, an essentially normal spectrum was obtained. The signal increase measured in our untreated patients is probably strong enough to be utilized in monitoring treatment in humans. Further studies should be performed to evaluate the effect of BMT (or ERT) in patients being younger at the initiation of treatment.

Acknowledgements We would like to thank the European Union HUE-MAN consortium for their cooperation.

Open Access This article is distributed under the terms of the Creative Commons Attribution Noncommercial License which permits any noncommercial use, distribution, and reproduction in any medium, provided the original author(s) and source are credited.

\section{References}

Autti T, Joensuu R, Aberg L (2007) Decreased T2 signal in the thalami may be a sign of lysosomal storage disease. Neuroradiology 49:571-578

Berg T, Hopwood JJ (2002) Alpha-Mannosidosis in the guinea pig: cloning of the lysosomal alpha-mannosidase cDNA and identification of a missense mutation causing alpha-mannosidosis. Biochim Biophys Acta 1586:169-176

Danielsen ER, Ross B (1999) Magnetic resonance spectroscopy diagnosis of neurological diseases. Marcel Dekker INC, New York

D'Hooge R, Lullmann-Rauch R, Beckers T, Balschun D, Schwake M, Reiss K, von Figura K, Saftig P (2005) Neurocognitive and psychotiform behavioral alterations and enhanced hippocampal long-term potentiation in transgenic mice displaying neuropathological features of human alpha-mannosidosis. J Neurosci 25:6539-6549

Grewal SS, Shapiro EG, Krivit W, Charnas L, Lockman LA, Delaney KA, Davies SM, Wenger DA, Rimell FL, Abel S, Grovas AC, Orchard PJ, Wagner JE, Peters C (2004) Effective treatment of alpha-mannosidosis by allogeneic hematopoietic stem cell transplantation. J Pediatr 144:569-573

Gutschalk A, Harting I, Cantz M, Springer C, Rohrschneider K, Meinck H-M (2004) Neurology 63:1744-1746

Johanson CE, Stopa EG, McMillan PN (2011) The bloodcerebrospinal fluid barrier: structure and functional significance. Methods Mol Biol 686:101-131

Magnitsky S, Vite CH, Delikatny EJ, Pickup S, Wehrli S, Wolfe JH, Poptani H (2010) Magnetic resonance spectroscopy of the occipital cortex and the cerebellar vermis distinguishes individual cats affected with alpha-mannosidosis from normal cats. NMR Biomed 23:74-79

Michalski JC, Haeuw JF, Wieruszeski JM, Montreuil J, Strecker G (1990) In vitro hydrolysis of oligomannosyl oligosaccharides by the lysosomal alpha-D-mannosidases. Eur J Biochem 189:369-379 
Ockerman PA (1967) A generalised storage disorder resembling Hurler's syndrome. Lancet 2:239

Patay Z (2005) Diffusion-weighted MR imaging in leukodystrophies. Eur Radiol 15:2284-2303

Patlas M, Shapira MY, Nagler A, Sheffer R, Gomori JM (2001) MRI of mannosidosis. Neuroradiology 43:941-943

Roces DP, Lullmann-Rauch R, Peng J, Balducci C, Andersson C, Tollersrud O, Fogh J, Orlacchio A, Beccari T, Saftig P, von Figura K (2004) Efficacy of enzyme replacement therapy in alpha-mannosidosis mice: a preclinical animal study. Hum Mol Genet 13:1979-1988

Seto T, Kono K, Morimoto K, Inoue Y, Shintaku H, Hattori H, Matsuoka O, Yamano T, Tanaka A (2001) Brain magnetic resonance imaging in 23 patients with mucopolysaccharidoses and the effect of bone marrow transplantation. Ann Neurol 50:79-92

Stinchi S, Lullmann-Rauch R, Hartmann D, Coenen R, Beccari T, Orlacchio A, von Figura K, Saftig P (1999) Targeted disruption of the lysosomal alpha-mannosidase gene results in mice resembling a mild form of human alpha-mannosidosis. Hum Mol Genet 8:1365-1372
Takahashi Y, Sukegawa K, Aoki M, Ito A, Suzuki K, Sakaguchi H, Watanabe M, Isogai K, Mizuno S, Hoshi H, Kuwata K, Tomatsu S, Kato S, Ito T, Kondo N, Orii T (2001) Evaluation of accumulated mucopolysaccharides in the brain of patients with mucopolysaccharidoses by (1)H-magnetic resonance spectroscopy before and after bone marrow transplantation. Pediatr Res 49:349-355

Vedolin L, Schwartz IV, Komlos M, Schuch A, Puga AC, Pinto LL, Pires AP, Giugliani R (2007) Correlation of MR imaging and MR spectroscopy findings with cognitive impairment in mucopolysaccharidosis II. AJNR Am J Neuroradiol 28:10291033

Walkley SU, Thrall MA, Dobrenis K, Huang M, March PA, Siegel DA, Wurzelmann S (1994) Bone marrow transplantation corrects the enzyme defect in neurons of the central nervous system in a lysosomal storage disease. Proc Natl Acad Sci USA 91:2970-2974

Wall DA, Grange DK, Goulding P, Daines M, Luisiri A, Kotagal S (1998) Bone marrow transplantation for the treatment of alphamannosidosis. J Pediatr 133:282-285 